\title{
L'Europe centrale dans le contexte de la géographie littéraire et symbolique
}

\section{Miloš Zelenka}

Traducteur : Marie-Odile Thirouin

\section{OpenEdition \\ Journals}

Édition électronique

URL : http://journals.openedition.org/recherchestravaux/523

DOI : 10.4000/recherchestravaux.523

ISSN : 1969-6434

\section{Éditeur}

UGA Éditions/Université Grenoble Alpes

\section{Édition imprimée}

Date de publication : 15 mai 2012

Pagination : 121-140

ISBN : 978-2-84310-227-1

ISSN : 0151-1874

\section{Référence électronique}

Miloš Zelenka, «L'Europe centrale dans le contexte de la géographie littéraire et symbolique »,

Recherches \& Travaux [En ligne], 80 | 2012, mis en ligne le 15 novembre 2013, consulté le 08 septembre 2020. URL : http://journals.openedition.org/recherchestravaux/523 ; DOI : https://doi.org/10.4000/ recherchestravaux.523 
Miloš Zelenka

Prague - České Budějovice

(Traduit du tchèque par Marie-Odile Thirouin)

\section{L'Europe centrale dans le contexte de la géographie littéraire et symbolique}

Ces vingt dernières années, le thème de l'«Europe centrale» a fait l'objet d'un regain d'interprétations, dues pour l'essentiel à un discours intellectuel qui évoque avec nostalgie la vision multiculturelle de la monarchie habsbourgeoise de la fin du $\mathrm{XIX}^{\mathrm{e}}$ siècle ${ }^{\mathrm{I}}$. Le souvenir d'un destin commun est venu corriger les réminiscences historiques renvoyant à la fameuse "prison des peuples", et sous son influence, la réalité de l'époque austro-hongroise s'est métamorphosée en photographie idyllique aux tons sépia, plus apte à refléter les humeurs subjectives et les idées préconçues que les conceptions et les projets politiques formulés avec netteté. Si, après I9I8, les changements institutionnels et géopolitiques avaient motivé ce type de discussions par la crainte d'un retour à l'ordre ancien, les conséquences de la Deuxième Guerre mondiale ont certes remis à l'ordre du jour l'idée d'une solidarité des divers États nationaux de la région, mais sous l'égide d'une doctrine monolithique qui s'est disloquée en 1989. Aujourd'hui, les débats politiques sur l'Europe centrale, qui a cessé de représenter une menace et un pion sur l'échiquier du pouvoir, s'attachent

I. Voir I. Pospíšil (éd.), Litteraria humanitas XI. Crossroads of Cultures : Central Europe. Kreuzwege der Kulturen: Mitteleuropa. Križovatky kultury: Stredni Evropa. Perekrestki kultury: Sredñaja Evropa, Brno, université Masaryk, 2002; H. Janaszek-Ivanickova et D. Fokkema (éd.), Post-modernism in Literature and Culture of Central and Eastern Europe, Katowice, Slask, 1996; P. Gerlich, K. Glass, B. Serloth (éd.), Mitteleuropäische Mythen und Wirklichkeiten. Ausformungen, Bedeutungen, Standortbestimmungen, Österreichische Gesellschaft für Mitteleuropäische Studien (coll. "Zentraleuropa/Mitteleuropa» vol. 6), Vienne, A. Marszałek, I996; I. Pospísil et M. Zelenka (éd.), Centrisme interlittéraire des littératures de l'Europe centrale, Brno, université Masaryk, I999. 
donc à construire une rhétorique au service d'une philosophie de la région, ainsi qu'une géographie littéraire et symbolique ${ }^{2}$.

L'Europe centrale s'est ainsi réellement métamorphosée dans le temps et dans l'espace, horizontalement et verticalement, mais aussi dans ce qui fait sa structure profonde. Il va de soi que la conception médiévale de l'Europe centrale est incompatible par exemple avec le nationalisme romantique du $\mathrm{XIX}^{\mathrm{e}}$ siècle; de même, les tournants historiques du $\mathrm{XX}^{\mathrm{e}}$ siècle que sont les années I9I8, I945 et I989, ont radicalement rompu avec la tradition antérieure prévalant sur le plan de l'apport spécifique de cette région à la culture, à la science, à la politique et sur le plan de ce qu'on peut en attendre pour l'avenir. Ainsi, hommes politiques, économistes, géographes, historiens et historiens de l'art définiront-ils et défendront-ils différemment la conception qu'ils en ont; les philologues ne s'entendront que difficilement avec les musicologues et les architectes sur la question de savoir quels sont les traits valides communs à tous les idéologèmes de la centre-européanité, de savoir où ils commencent et où ils finissent, et même de savoir si un problème comme celui-ci a sa place dans le discours scientifique. Le théoricien slovène de la littérature, Janko Kos, parle de différences essentielles entre conceptions littéraires, conceptions culturelles et historiques et enfin conceptions politiques qui, en règle générale, ne se recouvrent pas non plus avec la définition géographique de l'Europe centrales. En dépit de ces limites, le phénomène de l'Europe centrale qui, la plupart du temps, se manifeste sur le plan idéologique et politique et en même temps le déborde souvent en direction des domaines de la culture, de l'art, mais aussi de la sociologie, de la philosophie, de la sémiotique et des sciences voisines, reste une construction intellectuellement stimulante qui nous parle de nous-mêmes, c'est-à-dire des sources de notre identité et des racines de la civilisation européenne.

L'Europe centrale comme espace culturel et géographique ou comme carrefour entre Occident et Orient s'est toujours distinguée par la position variable de ses centres et de ses périphéries, fondamentalement instables, et par une interpénétration spécifique d'ethnies, de cultures et de religions. Le principe territorial du "contact" réciproque a conduit à un mode de communication plus intense et à l'échange de valeurs littéraires ainsi qu'à une compréhension plus profonde de celles-ci, mais aussi à leur confrontation et à des conflits de traditions artistiques et poétiques, de normes et de

2. Voir P. Demetz, Prague in Black and Gold: Scenes from the Life of a European City, New York, Hill \& Wang, I998

3. J. Kos, "Slovenska literatura in Srednja Evropa» («La littérature slovène en Europe centrale»), Slavistična revija, n I, vol. 38, Lubiana, I990, p. II-26. 
conventions ${ }^{4}$. La motivation métonymique de ce type de communication, découlant davantage d'un effet de "contiguïté» que d'une parenté génétique entre langues, peut évoquer non seulement le processus d'interculturalité décrit par Earl Miner', conséquence d'un horizon d'attente ouvert sur le plan de la réception (Hans Robert Jauss ${ }^{6}$ ), mais aussi une tentative en vue de postuler et de fixer le mythe littéraire de l'unité de divers ensembles et systèmes, en l'occurrence de l'Europe occidentale et de son origine civilisationnelle gréco-romaine. Le caractère hégémonique et universaliste de ce paradigme qui s'appuie sur l'idée de "grandes» littératures nationales «développées» et "dispensatrices d'influence», a été étayé par les modèles historiographiques des deux derniers siècles qui, avec leur «idéologie ethnocentrique», ont subrepticement diffusé le mythe de l'unité de la littérature de l'Europe occidentale et de son socle commun, sans prendre en considération la valeur d' "altérité" représentée par les littératures dites périphériques avec leurs racines distinctes7.

Le théoricien slovaque de la littérature Dionýz Durišin, en reconfigurant la littérature comparée traditionnelle, a recours à la géographie et à la géopolitique pour mieux saisir le processus d'échange interlittéraire européen, notamment dans l'aire littéraire centre-européenne. Par l'intermédiaire de l'analyse permise par les catégories clés qu'il a appliquées à la communauté interlittéraire (communauté séparée, communauté standard et communauté de centrisme interlittéraire), il parvient à formuler les lois d'une théorie de l'interlittérarité et aussi à comprendre le concept de littérature mondiale (universelle ${ }^{8}$ ). Les communautés interlittéraires représentent des phénomènes et des formations susceptibles de varier historiquement et structurés intérieurement qui, dans

4. Voir notre étude «K fenoménu středoevropských literatur (středoevropský literární prostor a jeho slovanský kontext)» ("Sur le phénomène des littératures centre-européennes [l'espace littéraire centre-européen et son contexte slave]»), dans M. Zelenka, Literární véda a slavistika (Science littéraire et slavistique), Prague, Academia, 2002, p. 59-79.

5. Voir E. R. Miner, Comparative Poetics : an Intercultural Essay on Theories of Literature, Princeton Universtiy Press, I990.

6. H. R. Jauss, Ästhetische Erfahrung und literarische Hermeneutik, Munich, Fink Verlag, 1977. Voir sur ce point P. Koprda, "Jaussova recepčna estetika a interkultúrne centrizmy (Medziliterárna komunikácia je otváranie sa prijímajúceho sebe)» («L'esthétique de la réception de Jauss et les centrismes interculturels [la communication interlittéraire est ouverte et réceptive]»), Slovak Review, n 2, vol. 8, Bratislava, I999, p. I53-168.

7. Voir F. Sinopoli, Il mito della letteratura europea, Rome, Meltemi, I999.

8. D. Durišin, Čo je to svetová literatúra? (Qu'est-ce que la littérature mondiale), Bratislava, Obzor, 1992; Teória medziliterárneho procesu I (Théorie du processus interlittéraire I), Bratislava, Ústav svetovej literatúry SAV, 1995; (et al.), Osobitné medziliterárne spoločenstvá I-6 (Communautés interlittéraires particulières, vol. I à 6), Bratislava, Veda, 1987-1993; (et al.), Medziliterárny centrizmus stredoeurópskych literatúr (Centrisme interlittéraire des littératures centre-européennes), České Budějovice (Jihočeská univerzita, Pedagogická fakulta) et Brno (Ústav slavistiky Filozofické fakulty Masarykovy univerzity), I998. 
leur développement historique, ont subi l'influence de divers éléments déterminants. Le premier de ces éléments à citer est le principe d'appartenance ethnique qui a servi à marquer l'analogie ou la parenté des différentes littératures nationales et de leurs sous-systèmes. Du principe ethnique dépend ensuite étroitement le facteur de la langue qui, par le passé, a pu non seulement relier divers ensembles littéraires entre eux, mais en même temps les différencier radicalement - le monolinguisme des littératures anglophones concerne par exemple plus de littératures nationales qui sont pourtant dissemblables sur le plan typologique et qui, pour les raisons les plus variées, sont devenues l'élément porteur de nombreuses communautés interlittéraires ou d'un centrisme interlittéraire (par exemple selon le lien des littératures coloniales avec la métropole d'origine). L'aspect géographique conduit explicitement à «dissocier» clairement différentes "sphères» littéraires en Europe (zones, régions, aires, et ainsi de suite) et à réaliser sur le plan pratique une carte littéraire de l'Europe qui comprend une partie méditerranéenne (médiévale), une partie centre-européenne, une partie orientale, une partie balkanique, une partie nordique, et ainsi de suite.

Parmi les autres critères introduits par Dionýz Ďurišin dans sa systématisation, on trouve par exemple les facteurs administratifs et institutionnels, la question confessionnelle et aussi le critère de différenciation évolutive. Pour résumer ce dernier, on peut dire qu'il s'agit là de savoir si un ensemble littéraire concret se développe en position dominante ou subordonnée et dans des conditions historiquement favorables ou défavorables à sa formation et à son développement. C'est que le partage fréquent des littératures européennes en "grandes» et «petites» littératures, en littératures «développées» ou "non développées», "dispensatrices d'influence» ou "soumises à influence», «légiférantes» ou "non légiférantes» en matière littéraire, a découlé du présupposé erroné selon lequel le développement des diverses littératures nationales serait uniforme. Or, l'une des lois principales auxquelles obéit entre autres le processus interlittéraire européen, c'est l'existence d'un développement non uniforme qu'on ne peut pas expliquer par une dépendance comprise de façon positiviste ou par une influence unilatérale. Il nous faut dans ce domaine tenir compte aussi de tout le point de vue politique et idéologique, qui fait par exemple que nous avions encore récemment à l'esprit un partage de l'Europe centrale en bloc «socialiste» et en bloc «non socialiste», partage qui correspondait dans le discours politique à la division géographiquement imprécise de l'aire centre-européenne en sphère d'influence "orientale» (soviétique), et en sphère d'influence "occidentale» (américaine).

Dans le discours habituel des politologues et publicistes, il est ainsi possible - en simplifiant consciemment - de modéliser et donc de distinguer 
deux conceptions de l'Europe centrale, l'une minimaliste et l'autre maximaliste ${ }^{9}$. La conception minimaliste considère cet espace comme la dernière "avancée» de l'Occident vers l'Est en matière de transmission des structures historiques et des valeurs culturelles; la centre-européanité devient dans ce contexte une position civilisatrice privilégiée qui regarde, dans l'optique de l'Europe de l'Ouest, à la fois en direction des Balkans «attardés" sur le plan du développement et en direction de la Russie, c'est-à-dire vers les parties méridionale et orientale de l'Europe ${ }^{\mathrm{I}}$. Cette conception minimaliste procède par oppositions binaires au sens de Jacques Derrida (nous et eux, à nous et aux autres, civilisation et barbarie) et a recours au mythe du centre et de la périphérie, de la frontière et de la fin de l'Europe, mythe qui contribue à une conception dichotomique (dualiste) de l'Europe centrale. C'est de cette manière que la comprend Milan Kundera lui-même qui, au milieu des années I980, a défini cet espace comme la région spécifique des petites nations prises entre l'Allemagne et la Russie, région qui appartient culturellement à l'Occident, mais est politiquement rangée (depuis 1945) du côté de l'Est ${ }^{\mathrm{II}}$.

La conception maximaliste, quant à elle, envisage l'Europe centrale de façon essentiellement axiologique : les constructions géographiques concrètes sont ici une production secondaire, et l'existence même du centre de l'Europe est mise en question, de même que parfois la dissociation fonctionnelle entre parties séparées de l'Europe. L'Europe, y compris donc sa partie centrale, se définit dans ce contexte comme ensemble d'idées historiquement fondées, la plupart du temps en relation avec la tradition chrétienne latine, définissant le lien du vieux continent dans son ensemble avec les continents voisins, surtout avec l'Asie dont l'Europe est issue sur le plan civilisationnel, comme nous l'oublions parfois. L'historien hongrois László Kontler donne à cette thèse la forme concrète suivante :

À côté de la vision de l'Europe centrale comme utopie [...], il existe une Europe centrale qui s'exprime en concepts analysables objectivement $[\ldots]:$ partage du pouvoir entre la couronne et les états ou les ordres dotés de privilèges, hiérarchie féodale fondée sur une assise légale, villes libres et répartition des tâches entre ces villes et leur environnement agraire, culture chevaleresque, humanisme de la Renaissance et Réforme ${ }^{12}$.

9. Voir L. Kontler, «Úvahy o symbolické geografii» («Réflexions à propos de la géographie symbolique»), dans Dějiny Mad’arska (Histoire de la Hongrie), Prague, Nakladatelství Lidové noviny, 200I, p. IO-II.

Io. I. Dorovský, Balkán a Mediterán. Literárnè historické a teoretické studie (Les Balkans et la Méditerranée. Étude théorique et d'histoire littéraire), Brno, université Masaryk, 1997.

II. M. Kundera, "Un Occident kidnappé ou la tragédie de l'Europe centrale», Le Débat, $\mathrm{n}^{\circ} 27$, novembre 1983, p. 3-22.

I2. L. Kontler, art. cité, p. II-I2. 
Bien que, dans cette conception maximaliste, l'Europe centrale n'ait pas de limites strictes ni de forme fixe, on voit bien qu'elle se rattache plutôt à l'Occident dont elle fait partie intégrante - une partie certes attardée et moins développée, mais où, à la différence de ce qui se passe dans la civilisation musulmane ou byzantine, «l'aspect de développement produit par la pénétration des structures occidentales vers l'Est ${ }^{13}$ » arrive à accomplissement.

Revenons à la conception minimaliste : les racines de cette façon de voir remontent loin, jusqu'au Moyen Âge, quand l'Europe centrale se confondait avec la monarchie danubienne qui se constitua définitivement au XVII ${ }^{\mathrm{e}}$ siècle en réunion de nations dirigée contre la menace turque (par exemple lors de la victoire de 1683 sur les Turcs à Vienne); de même, au XIX ${ }^{e}$ siècle, la monarchie habsbourgeoise avait à protéger cette zone contre l'expansion russe en direction de l'Occident. De là venaient les craintes que l'Autriche-Hongrie ne se disloque, elle qui était considérée comme un élément stabilisateur pour l'équilibre centre-européen (chez l'historien tchèque du XIx siècle František Palacký par exemple). Au cours de la Première Guerre mondiale, le militariste prussien Friedrich Naumann donna à cette conception un aspect théorique en l'intégrant à la propagande allemande de guerre, s'efforçant de créer une monarchie centre-européenne sous la direction de l'Allemagne ${ }^{14}$. À la fin du $\mathrm{xx}^{\mathrm{e}}$ siècle encore, l'historien et publiciste français d'origine hongroise, François Fejtö, exprimait son regret devant le fait que l'éclatement de l'Autriche-Hongrie ait signifié une catastrophe pour l'histoire politique de l'Europe centrale, estimant que nous en supportons les conséquences jusqu'à aujourd'hui ${ }^{15}$, ce qui signifie que sans l'éclatement arbitraire de la monarchie austro-hongroise et sa dislocation nullement nécessaire, on n'en serait pas arrivé à Yalta ni à la soviétisation de l'espace centre-européen ni, par exemple, au début des années I990, à la partition de la Tchécoslovaquie ou encore au conflit sanglant entre les nations de l'ancienne Yougoslavie. Pour Fejtö, l'Europe centrale est «le symbole d'une occasion manquée ${ }^{16} »$, surtout du fait qu'en 1918 , elle a donné la priorité aux nationalistes et non au modèle fédéraliste d'organisation des relations politiques mutuelles. Bien que l'historien français néglige ici entièrement le droit naturel des petites nations slaves à l'autodétermination qui, dans cet espace, constitue l'aboutissement logique de processus historiques de longue durée tendant à l'émancipation et à la création d'États indépendants, il perçoit relativement bien l'essor, sur le territoire de la monarchie

13. Ibid., p. I2.

I4. Voir l'ouvrage de Fr. Naumann, Mitteleuropa, Berlin, Reimer, I9I5.

I5. Fr. Fejtö, Requiem pour un empire défunt. Histoire de la destruction de l'Autriche-Hongrie, Seuil, I993, p. I7-I8 (la traduction tchèque date de 1998).

16. Ibid., p. 377. 
habsbourgeoise, d'une conscience centre-européenne se constituant au fil des siècles et survivant jusqu'à l'époque contemporaine, conscience d'une sorte d'Europe centrale en petit qui a représenté un territoire unifié administrativement et économiquement avec une forte urbanisation et, surtout, un carrefour réel de nations avec une culture bigarrée. Ainsi l'Autriche-Hongrie a-t-elle incarné un espace socioculturel doté d'un fort sentiment de solidarité supranational (mais aussi d'une forte haine mutuelle) où s'est constitué le type humain de l'"homo habsburgiensis" dont la sensibilité et la mentalité étaient autres que celles des grandes nations de l'Occident et de l'Orient, des Français, des Allemands, des Anglais et des Russes ${ }^{17}$.

Si la «variante allemande» de cette conception, issue du point de vue du militariste prussien Naumann, a été plus agressive et plus visible politiquement (elle a atteint un sommet de compromission avec la tentative hitlérienne d'organisation du soi-disant "espace de l'Est» ou Ostraum), l'Europe centrale "autrichienne», même après la dislocation de l'Autriche-Hongrie, a donné lieu à une discussion intellectuelle, notamment dès les années I980 où elle a été essentiellement déclenchée par les émigrants et les dissidents des pays d'Europe centrale : un rôle essentiel a été joué ici par l'article de Milan Kundera intitulé "Únos Západu» ("Rapt de l'Occident») ${ }^{18}$ »; ce texte exprime le scepticisme de Kundera quant aux solutions politiques et idéologiques réglant l'organisation étatique en Europe centrale, un espace qui, pour lui, n’a pas de frontières géopolitiques nettes, mais est plutôt un lieu de confrontation, de dialogue et de coexistence, de mémoire culturelle et d'expérience historique sur le mode analogique. Des textes signés de Václav Havel, György Konrád et Czesław Miłosz, par exemple, ont aussi approuvé l'existence d'une identité centre-européenne comme tradition spirituelle authentique niant la réalité géopolitique imposée après $1945^{19}$.

I7. Ibid.

18. M. Kundera, "Únos Západu", I50 ooo slov, IO.I.1985, p. II2-II9; en anglais : "The Tragedy of Central Europe", The New York Times Review of Books, 26 avril 1984, p. 33-37 (le même texte est publié sous le titre "A Kidnapped West or Culture Bows Out», Granta, n ${ }^{\circ}$ II, I984, p. 95-II8).

19. V. Havel, "Anatomie jedné zdrženlivosti " ("Anatomie d’une réticence») 1985; G. Konrád, Antipolitika (Antipolitique) 1984; C. Miłosz, Świadectwo poezji (Témoignage de la poésie) I983. Ces textes sont interprétés dans le détail par C. Balabanová dans «Stredná Európa a stredoeurópsky geografický kultúrny priestor (S prihliadnutím na interpretácie Milana Kunderu, Györgya Konráda, Václava Havla, Czesława Miłosza)» («L’Europe centrale et l'espace culturel géographique centre-européen [en tenant compte de l'interprétation de M. Kundera, de G. Konrád, de V. Havel et de C. Miłosz]»), dans Hrdina v stredo-európskych a balkánskych literatúrach I9. a 20. storočia (Le héros dans les littératures centre-européennes et balkaniques des $X I X^{e}$ et $X X^{e}$ siècles), Bratislava, Veda, 2004, p. 9-19. 
Restons-en au texte de Kundera : son mérite vient de ce qu'il a détourné la discussion sur l'Europe centrale du discours politologique pour la placer à un niveau philosophique et littéraire et de ce que ses réflexions, qu'elles aient suscité l'assentiment ou le rejet, sont devenues un modèle de réflexion initial qu'il est impossible de négliger. Bien qu'on puisse identifier chez Kundera une secrète nostalgie de l'atmosphère spirituelle perdue de la monarchie habsbourgeoise, nous tenons là la stricte négation de la variante "allemande» de l'Europe centrale. Selon lui, celle-ci n'existe plus en tant que telle sur le plan géopolitique et n'a ni centre clair ni frontières; leur variabilité et leur inventivité dessinent un territoire marqué par un destin historique commun et caractérisé par un lien analogique avec des valeurs culturelles partagées qui donnent forme aux petites nations situées dans l'espace flou séparant l'Allemagne de la Russie. Pour Kundera, jamais ces petites nations n'ont été conquérantes, et leur existence a toujours dépendu de la volonté de leurs puissants voisins, une petite nation ayant toujours conscience qu' elle peut disparaître. Ethniquement, l'Europe centrale représente un mélange multinational complexe, slave et non slave, avec un élément juif original qui symbolise l'expérience tragique de l'intellectuel centre-européen. Au fond, Kundera assimile et identifie l'idéal d'organisation géopolitique, sociale et spirituelle de l'Europe centrale avec un modèle fédératif, avec une communauté démocratique de petites nations ayant une valeur universelle sur le plan culturel - cette intégration dans un ensemble supranational n'élimine toutefois pas la personnalité de chacun de ses membres, centres locaux enrichissant l'héritage commun. L'Europe centrale a en outre toujours rempli la fonction de "pont» et de voie de liaison : sur ce point, Kundera renoue librement avec le sentiment austroslaviste de František Palacký et avec les réflexions politiques que le premier président de la République tchécoslovaque, Tomáš Garrigue Masaryk, a consacrées à la fonction stratégique de l'Europe centrale comme axe symbolique assurant la stabilité du système de sécurité européen après la Première Guerre mondiale. Selon Kundera, c'est justement l'Europe centrale qui, par sa position médiane au "cœur» de l'organisme européen, doit équilibrer ses extrémités occidentale (le pouvoir technologique) et surtout orientale (le totalitarisme soviétique). Il n’est donc pas étonnant de voir Kundera exclure radicalement la Russie de l'Europe à cause de son passé non démocratique. Il cite par ailleurs, comme traits distinctifs propres au centreeuropéanisme, le scepticisme et l'(auto-)ironie qui permettent à celle-ci de dépasser ses doutes existentiels quant à sa place dans la "grande» histoire et, surtout, son manque de confiance dû à sa petitesse. Ces éléments, Kundera les trouve directement élaborés en stratégies d'auteur, sous les espèces de techniques narratives, de structures romanesques et de formes artistiques présentes 
chez les écrivains et les artistes typiquement centre-européens (Béla Bartók, Hermann Broch, Witold Gombrowicz, Jaroslav Hašek, Leoš Janáček, Franz Kafka, Robert Musil, Bruno Schulz, Stanisław Ignacy Witkiewicz, etc.) qu’il mentionne entre autres dans son Art du roman [1986].

Si l'Europe centrale de Kundera ne représente pas un objet géographiquement stable avec des frontières claires, la communauté dynamique et historiquement changeante des petites nations ne restant jamais identique à elle-même au cours de l'histoire, pour son collègue tchèque Václav Havel, il n'est pas non plus question de dénomination précise, mais plutôt de force de l'idée, de mécanismes de fonctionnement et de critères au moyen desquels aborder ce problème. Havel, dans son essai intitulé "Anatomie jedné zdrženlivosti» (Anatomie d'une réticence) [1985], compare l'Europe centrale à "un phénomène spirituel, culturel et mental» que "façonnent à chaque instant des expériences historiques spécifiques précises ${ }^{20} »$. Comme signes distinctifs essentiels du substrat psychique centre-européen tel qu'il est présent dans la mémoire culturelle commune, Havel cite le scepticisme, le sens de l'ironie, l'auto-ironie dont font partie de l'humour noir et surtout les rencontres génériques d'éléments comiques et tragiques à l'intérieur d'un ensemble original, comme il en trouve, dans des configurations diverses, chez Franz Kafka, Jaroslav Hašek, Robert Musil ou Ludvík Vaculík (Český snár [ La Clef des songes]). C'est précisément à la littérature, avec sa formidable capacité de réception - on peut remarquer ici à quel point l'auteur dépend directement de l'idéologie de la Renaissance nationale et de sa foi dans le pouvoir des mots -, de devenir la forme naturelle et le réceptacle des archétypes et des attitudes hérités de l'histoire, comme par exemple un scepticisme lentement élaboré au fil des siècles ou encore la défiance à l'égard des idéologies et des projets utopiques.

Chez Havel et ses collègues intellectuels, l'Europe centrale est envisagée comme centre culturel mythique de toute l'Europe, occupé par les petites nations et balloté au gré des intérêts géopolitiques des grandes puissances. Havel, à la différence de la conception qu'on trouve chez Kundera d'un espace polycentrique, aux frontières mouvantes et multinational sur le plan de l'histoire culturelle, situe le centre géographique de l'Europe centrale dans les pays tchèques qui, selon lui, auraient toujours été un carrefour pour les courants, les impulsions et les initiatives les plus variées. Dans la vision de Havel, qui critique la division de l'Europe en deux blocs opposés, l'Europe

20. V. Havel, Do rüzných stran. Eseje a články z let 1983-1989 (Dans diverses directions. Essais et articles des années 1983-1989), V. Prečan (éd.), Prague, Lidové noviny, 1989, p. 74. Pour la traduction française de cet essai, voir V. Havel, Essais politiques rassemblés par R. Errera et J. Vladislav, Seuil, I99I. 
centrale devient l'idéal d'une Europe unifiée démocratiquement comme communauté fraternelle d'États libres et souverains. On reconnaît ici l'éthos moral de la société civile, la perspective anthropologique et l'ancrage historique et philosophique chers à Havel, quoiqu'un léger déplacement s'opère dans sa rhétorique postérieure à 1989. Dans ses discours politiques du début des années 1990, faits en qualité de chef d'État, Havel glisse en effet vers l'idéalisme quand il constate qu'aujourd'hui existe la possibilité de «transformer l'Europe centrale, phénomène jusqu'à présent essentiellement historique et spirituel, en phénomène politique ${ }^{2 \mathrm{I}} »$. Bien que l'idée soit toujours présente d'une Europe qui ne doit pas être une collection d'États, de nations, de cultures, d'ethnies et de régions coexistant les uns à côté des autres, mais bien un espace spirituel commun fondé sur le principe d'une société ouverte (ce qui implique démocratie, respect des droits de l'homme, marché libre, propriété privée, pluralisme politique, etc.), ce processus doit pourtant se dérouler, pour Havel, sous les auspices des mécanismes intégrateurs ouesteuropéens qui s'étendront à l'Europe centrale, puis à l'Europe orientale, si possible sous la forme d'États Unis d'Europe ou de confédération européenne (François Mitterrand). Havel a ainsi considéré l'adhésion des petites nations centre-européennes à l'OTAN et à l'Union européenne, au moment du changement de millénaire, comme l'accomplissement d'une vision de l'Europe centrale libre, et dans le cas particulier des pays tchèques, comme le point final mis à la discussion, permanente ici, sur le sens de l'histoire nationale.

L'ancien dissident hongrois György Konrád, avec sa conception d'une politique antipolitique, est proche des positions de Havel : comme lui, il relie la centre-européanité à un mode de pensée sceptique et auto-ironique; mais l'adhésion à l'identité centre-européenne représente avant tout pour Konrád le prolongement et l'aboutissement spécifique de la tradition et de la culture nationales hongroises qui, pendant mille ans, se sont orientées culturellement et politiquement par rapport à l'Occident avant de prendre leur essor au cours de la seconde moitié du XIx siècle, dans le cadre de l'Autriche-Hongrie dualiste $^{22}$. Si, après 1945, l'hégémonie soviétique a été la cause de la tragédie de l'Europe centrale, le centre-européanisme devient, dans l'argumentation de Konrád, l'expression d'une conscience collective qui, historiquement, a toujours tiré ses racines de sa parenté avec l'Occident. De même, l'écrivain polonais et lauréat du prix Nobel de littérature, Czesław Miłosz, dans son

2I. V. Havel, Projevy (leden-červen I99o) (Discours [janvier-juin I9go]), V. Prečan (éd.), Prague, Nakladatelství Vyšehrad, I990, p. 43.

22. G. Konrád, Antipolitik. Mitteleuropäische Meditationen, Francfort-sur-le-Main, Suhrkamp, 1985 (en français : L’Antipolitique, La Découverte, I987). 
recueil d'essais intitulé Témoignage de la poésie [1983] et dans Stredoevropské postoje (Positions centre-européennes) [1986], déplace l'Europe centrale vers l'est, en direction de l'Estonie et de la Lituanie (Miłosz est d'ailleurs natif de Vilnius), et il fait coïncider leur ligne de démarcation avec la fracture religieuse de l'Europe entre christianisme occidental latin et christianisme oriental (orthodoxe). L'Europe centrale est partout là où l'on trouve une dépendance culturelle directe vis-à-vis de la civilisation occidentale antique sous la forme de la tradition culturelle gréco-romaine. Celle-ci déborde l'espace de la monarchie habsbourgeoise, tant sur le plan de la géographie que des idées, et englobe finalement jusqu'à l'ancien État médiéval polono-lituanien (Rzeczpospolita). Du reste, le mythe d'une union fédérative de nations sous domination polonaise se rencontre dans la littérature et la publicistique polonaises depuis fort longtemps et jusqu’à aujourd'hui. Il suffit de rappeler ici la vision esthétique de Sienkiewicz d'une démocratie nobiliaire rassemblant Polonais, Ukrainiens, Biélorusses et Lituaniens sous la houlette d'un État polonais $^{23}$. Miłosz n’hésite pas, dans ce contexte, à rappeler des exemples de collaboration tchéco-polonaise de l'époque de la Réforme et de l'humanisme tardif, c'est-à-dire de l'époque où s'impose peu à peu en Europe centrale le style baroque universel et typologiquement homogène en dépit des variantes nationales.

Il va de soi que Miłosz, par son attitude de refus à l'égard de la Russie et par sa réticence à définir un centre et des frontières pour l'Europe centrale, se situe plus près de Kundera que de Havel. Cela ressort entre autres de son ouvrage Rodzinna Europa (Une autre Europe) [1959] dans lequel Miłosz oppose l'Europe "privée» (c'est-à-dire la petite Europe natale où je suis né) et l'Europe "publique» (la grande Europe) : les limites géographiques et le centre réel comme le centre mythique de l'Europe sont ici encore plus dilués. La centre-européanité représente pour lui une valeur symbolique saisie par l'intermédiaire d'une stratégie d'auteur spécifique qui n'est pas seulement documentaire ou autobiographique, mais a recours à la fable et à la fiction : l'Europe centrale est un code génétique inscrit dans nos formules comportementales et nos modèles communicationnels; ce sont nos souvenirs, notre mémoire subjective, mais aussi les destins réels de personnes concrètes représentant sur un mode synecdochique les péripéties et les catastrophes connues par les petites nations opprimées.

23. A. Měšt'an, "Der mitteleuropäiche Raum in den Literaturen Mitteleuropas nach I945" ("L’espace mitteleuropéen dans les littératures de la Mitteleuropa après 1945»), dans Westmitteleuropa, Ostmitteleuropa - Vergleiche und Beziehungen. Festschrift für Ferdinand Seibt zum 65. Geburtstag, W. Eberhard, H. Lemberg, H.-D. Heimann, R. Luft (éd.), Munich, Oldenbourg, 1992, p. 369-376. 
On trouve une démarche similaire, issue d'une sorte d'anthropologie culturelle ou bien d'une microhistoire de type postmoderne, chez le romancier polonais appartenant à la génération intermédiaire, Andrzej Stasiuk, qui fait de l'Europe centrale une histoire privée, individuelle et vivante, un épisode subjectif. Celui-ci peut se dérouler par exemple à l'occasion d'un dialogue entre personnes, ou il peut surgir sous forme d'émotion indéfinissable qui, selon les présupposés du postmodernisme, se répète sans cesse à l'occasion d'actes quotidiens relevant de notre communication avec notre environnement. Ce monde propre à un héros modeste dépourvu de pathétique, monde qu'il connait intimement et dont il ne peut s'évader, on le trouve également chez le romancier tchèque Bohumil Hrabal dont les "palabreurs" passent par les traumatismes de l'identité centre-européenne (peur, angoisse, déracinement, tentative de changement suivie de rechutes perpétuelles). Le romancier ukrainien Yuri Andrukhovych, qui a écrit en commun avec Andrzej Stasiuk un essai intitulé Moja Europa. Dwa eseje o Europie zwanej Środkowej (Mon Europe. Deux essais sur l'Europe dite centrale ${ }^{24}$ ), relie quant à lui l'Europe centrale au motif du voyage : les destins privés des personnes vivant dans le vaste espace situé entre l'Allemagne et la Russie sont défigurés par la grande Histoire, et pourtant, en guise de défense, nous nous en accommodons avec le sentiment qu'en tant qu'individus, nous ne pourrons de toute façon jamais la tourner à notre avantage - la véritable Europe centrale, c'est le monde que je connais intimement, il n'a pas de centre ni de limites, il se trouve là jusqu'où on peut aller, jusqu'où on peut toucher, jusqu'où on peut voir.

Souvent, les réflexions sur l'Europe centrale convoquent dans la géographie symbolique le Danube comme élément à la fois diviseur et unificateur. Le germaniste italien Claudio Magris, par exemple, envisage le motif de la centre-européanité en lien avec une enquête sur le mythe habsbourgeois, avec la sacralisation du Danube qui franchit les frontières de l'Europe centrale et la relie aux Balkans et à l'espace méditerranéen :

Le Danube, c'est [l'Europe centrale] germano-magyaro-slavo-[romano-juive], [située à l'extrême opposé du] Reich germanique, [c'est] l'œcuménisme "hinternational», [c'est] un monde [situé «derrière» les nations] ${ }^{25}$.

24. J. Andruchowycz, Środkowoschodnie rewizje (Remix centre-européen), dans J. Andruchowycz, A. Stasiuk, Moja Europa. Dwa eseje o Europie zwanej Środkowej (Mon Europe. Deux essais sur l'Europe dite centrale), Wołowiec, Czarne, 200I, p. 7-74 (en français: Y. Andrukhovych, A. Stasiuk, Mon Europe, ouvr. cité).

25. Cl. Magris, Danubio, Garzanti, I986; en français : Danube, Gallimard, I988, p. 38). (NdT : la traduction tchèque date de 1992 et a servi de base aux modifications ici introduites dans le texte.) 
Le Danube, fleuve sacré des Slaves sur toute sa longueur, c'est la frontière entre l'Europe et les Balkans, nous dit Magris; et tandis que le Rhin des Allemands veille sur la pureté de la race germanique (pensons à la lourde mystique des opéras de Wagner), le Danube signifie la communication et le dialogue : par son intermédiaire, Allemands et Autrichiens sont en contact avec les Slaves de l'Ouest, mais aussi avec les Hongrois et les Slaves du Sud, ainsi qu'avec les musulmans et avec la culture orthodoxe.

Bien que le motif du Danube, quand il est en rapport avec le mythe habsbourgeois, exprime la nostalgie de l'empire multinational et d'une identité centre-européenne de type supérieur, les opinions divergent quant à la question de savoir dans quelle mesure le centre-européanisme s'est reflété ou non au centre même de la monarchie, en Autriche. Tandis que Magris reste sceptique (il ne considère pas le centre-européanisme comme constante véritable de l'histoire autrichienne ${ }^{26}$ ), le germaniste russe Dmitrij Zatonskij parle quant à lui d'aire politico-culturelle danubienne dans laquelle l'Autriche, depuis sa position dominante, a joué un rôle de pont, c'est-à-dire de maillon intermédiaire entre l'Occident germanique et l'Orient slave, ou a éventuellement occupé la position de gardien de l'espace ouest-européen ${ }^{27}$ - en guise d'illustration, il suffit de paraphraser librement le mot ironique souvent cité du chancelier autrichien Metternich, selon lequel la frontière de l'Europe (il faut entendre : de l'Europe "civilisée») s'arrête aux portes de Vienne. Comme le montre la slaviste russe Svetlana Serlaimova, cette théorie néglige toutefois l'importance des contacts directs entre littératures slaves de l'Ouest qui, dans le cadre de la monarchie habsbourgeoise, ont constitué une communauté interlittéraire propre avec un ensemble complexe de liens internes et externes $^{28}$.

Quoique de façon générale, la civilisation autrichienne ait fait souvent partie des thèmes de prédilection des écrivains slaves (mais aussi hongrois et roumains), elle l'était alors à titre de cadre extérieur : c'est le choix de la langue, en tant que code distinctif et facteur d'identification, qui était alors déterminant, et ce choix, les écrivains slaves de l'époque de la Renaissance nationale l'ont fait le plus souvent en fonction de leur origine ethnique. Nous

26. Cl. Magris, Il mito absburgico nella letteratura austriaca moderna [1963], Milan, Einaudi, I988; en français : Le Mythe et l'empire dans la littérature autrichienne moderne, L'Arpenteur, I99I, p. 29 (la traduction tchèque date de 200I).

27. D. V. Zatonskij, Avstrijskaja literatura $v$ XX stoletii (La Littérature autrichienne au $X X^{e}$ siècle), Moscou, Izdat. Hudožestvennaja Literatura, 1985.

28. S. A. Serlaimova, «Slovanské literatúry v kontexte rakúsko-uhorskej štátnosti» («Les littératures slaves dans le contexte de l'État austro-hongrois»), dans D. Durišin, Osobitné medziliterárne spoločenstvá (Communautés interlittéraires particulières), vol. 5, Slovanské literatúry (Littératures slaves), Bratislava, Veda, I992, p. I67-I79. 
pouvons tirer une leçon instructive du domaine linguistique, de la théorie des familles de langues ou encore des aires dessinées par l'analyse des relations géographiques entre caractères typologiques de langues cohabitant dans un voisinage étroit (tout en étant structurellement distinctes ${ }^{29}$ ). Des influences et des contacts nombreux enrichissent mutuellement les langues, quoiqu'elles conservent leur autonomie de développement historique. La linguistique moderne parle par exemple d'aire linguistique centre-européenne, ou plus largement encore d'aire danubienne, où des langues indo-européennes issues de deux groupes distincts, germanique et slave, se sont trouvées en contact et en position d'influence mutuelle avec le hongrois de la famille des langues finno-ougriennes : elle attire ainsi l'attention sur les processus parallèles et les tendances convergentes dans le domaine de la phonologie et de la phonétique, mais aussi de la morphologie et surtout du lexique ${ }^{30}$.

Sur la question du centrisme centre-européen ou du concept même de Mitteleuropa, on a entendu s'exprimer au début des années I990 l'ancien Pragois et doyen des études littéraires mondiales René Wellek. Dans le premier des trois entretiens qu'il a accordés au germaniste américain d'origine tchèque, Peter Demetz, dans les pages du magazine Cross Currents, il se montre relativement sceptique quant à ce concept, dans le sens où il est en lui-même suspect, étant le produit des réflexions de Friedrich Naumann au cours de la Première Guerre mondiale ${ }^{31}$. Ce concept a fait partie (du moins selon Wellek) de la propagande de guerre allemande de l'époque et visait à la création d'une monarchie centre-européenne qui aurait été plus étendue que la Prusse. La conception même qu'on peut avoir de l'Europe centrale est selon Wellek assez vague, car les frontières ne sont pas claires d'une notion qui est plutôt le produit d'une humeur nostalgique. Après avoir parlé de

29. Le fondateur de la typologie linguistique par aires géographiques est le linguiste russe N. S. Troubetzkoï, en collaboration avec le structuraliste tchèque d'origine russe R. Jakobson. Leur conception est issue de la conviction que les langues, du fait de leur proximité géographique, s'influencent sur le plan communicationnel à tel point que leurs caractères structurels peuvent évoluer en commun.

30. Voir S. M. Newerkla, «Rozmanitost jazyků a kultur v Rakousku-Uhersku a jejich konvergentní tendence» ("La diversité des langues et des cultures en Autriche-Hongrie et leurs tendances convergentes»), dans I. Pospíšil et M. Moser (éd.), Comparative Cultural Studies in Central Europe, Ústav slavistiky Filozofické fakulty Masarykovy univerzity, 2004, p. II-42, et H. Kurzová, "Mitteleuropa als Sprachareal», («La Mitteleuropa comme aire linguistique»), Germanistica Pragensia, n ${ }^{\circ} 3$, Prague, 1998, p. 57-73.

3I. P. Demetz, "A conversation with René Wellek", Cross Currents. A Yearbook of Central European Culture, n 9, 1990, p. I35-I45. Du même : "Second Conversation with René Wellek", Cross Currents, no I0, I99I, p. 235-25I, et "Third Conversation with René Wellek», Cross Currents, $\mathrm{n}^{\circ}$ II, I992, p. 79-92. R. Wellek exprime ses opinions sur la centre-européanité essentiellement dans le premier entretien, réalisé en 1989. 
son propre père qui, fonctionnaire autrichien, a quitté Vienne pour s'installer à Prague et apporter ainsi par enthousiasme son soutien à la nouvelle République tchécoslovaque, Wellek répond de nouveau à la même question. Peter Demetz y revient en effet, un peu par provocation, en faisant valoir, à propos de la propre vie de René Wellek (écoles tchèque et allemande, Vienne, Prague, va-et-vient contradictoire entre Est et Ouest, Nord et Sud), à quel point celle-ci est mitteleuropäisch. Wellek admet alors qu'il est un CentreEuropéen lié étroitement au tchèque, à l'allemand et à l'anglais, mais en même temps, il fait remarquer que la vieille Mitteleuropa, celle qui a existé sous l'Autriche, n'est plus possible, ne serait-ce qu'en conséquence de l'invasion soviétique de l'année 1944 (l'entretien a été réalisé avant les changements considérables intervenus dans cet espace à la fin de l'année 1989).

Les deux participants à la discussion, Demetz comme Wellek, expriment donc leur scepticisme quant à la centre-européanité : en conclusion, Wellek assure qu'entre Lubiana, Prague, Trieste, Budapest et Vienne, il n'y a pas de communication intense même si, en même temps, il est d'avis que ces lieux sont réunis par leur position vis-à-vis de l'Occident. Entre les littératures slaves, par exemple à l'époque du réalisme, il n'y a pas selon lui de liens privilégiés réciproques, et les ressemblances entre elles viennent de ce que ce sont les mêmes littératures occidentales qui ont agi sur elles. Wellek, qui a été incontestablement influencé par la version anglaise de l'article de Kundera sur l'Europe centrale, néglige ici de parler de l'action incontestable et, sur bien des points, tout à fait dominante exercée par le réalisme russe (qui, il faut aussi l'avouer, a puisé à son tour à la source des littératures de l'Europe occidentale) : la réceptivité à l'égard des impulsions en provenance de Russie a été facteur à la fois de l'inspiration slave, d'aspects sociaux et d'une certaine poétique. Néanmoins, l'opinion de Wellek, pour qui l'unité culturelle des littératures slaves de l'Ouest ne découle nullement d'une communication réciproque, mais d'une position de type analogique par rapport aux centres culturels occidentaux, se rencontre fréquemment dans le milieu des slavistes français qui attirent l'attention sur l'importance de Paris comme "capitale" des cultures centre-européennes, en particulier pendant les années 1918-1939 au cours desquelles les formes d'échange intellectuel entre la France et les pays d'Europe centrale se sont renforcées ${ }^{32}$. Un certain nombre de slavistes français utilisent d'ailleurs le concept d' "Europe médiane», expression que nous pourrions paraphraser approximativement en parlant d'Europe «située entre slaves, 1997. 
plusieurs zones», «intermédiaire», «transitionnelle ${ }^{33}$ » : il s'agit là du concept d'Europe centrale considérablement élargi, avec un caractère maximaliste dont nous allons reparler. Les littératures centre-européennes ne sont pas assimilées ici de façon simpliste aux littératures slaves, mais comprises comme communauté culturelle de la zone intermédiaire entre Ouest et Est, devenue après I945 partie intégrante du bloc soviétique ${ }^{34}$.

Si les conceptions "minimalistes» issues du modèle habsbourgeois ou allemand amendé se sont développées à partir de la définition d'un centre géographique et de celle, relativement précise, de frontières et d'un territoire propre à l'Europe centrale, la conception «maximaliste», qui a évité de déterminer le centre géographique de l'Europe, a en fait identifié l'Europe centrale à l'Europe tout court : le concept d'Europe peut être compris non seulement dans un sens plus étroit, géographique, mais aussi dans un sens plus large, axiologique - dans ce cas, l'Europe n'apparaît pas seulement comme territoire et comme continent s'étendant de l'Atlantique et de la mer du Nord à l'Oural et à la mer Méditerranée, mais surtout comme «idée advenue dans l'histoire [...] en étant définie sur la base de valeurs en lien avec les autres continents ${ }^{35}$ ", c'est-à-dire comme revendication consciente de l'unité dans l'hétérogénéité et d'une vision du monde multiculturelle et multicivilisationnelle. Une telle conception peut fournir une réponse à la question de savoir si, par exemple, la littérature serbe, la littérature ukrainienne ou même la littérature roumaine font encore partie, par leur caractère, des littératures des nations centre-européennes. Car l'espace littéraire centre-européen forme, dans l'optique maximaliste, un complexe intégrateur slave, germanique (allemand et éventuellement autrichien), partiellement latin même (avec l'Italie du Nord et la Roumanie), finno-ougrien (avec la Hongrie) et juif, dont l'unité vient tantôt de la culture de langue allemande, tantôt d'une autre culture, slave souvent (dans le cas des pays tchèques, de la Pologne, de l'Ukraine) - complexe qui, d'un côté, apparaît comme un phénomène autonome et homogène, et de

33. Voir H. Voisine-Jechova, «Lidská identita a motiv toho, co nelze vyjádřit u Mickiewicze a Máchy v rámci slovanských literatur» ("Lidentité humaine et le motif de ce qu’on ne peut pas dire chez Mickiewicz et Mácha dans le cadre des littératures slaves»), dans I. Pospísil et M. Zelenka (éd.), Karel Krejči a evropské myšlení (Karel Krejči et la pensée européenne), Brno, Slavistická společnost Franka Wollmana, 2006, p. 70-7I.

34. Histoire littéraire de l'Europe médiane des origines à nos jours, M. Delaperrière (dir.), L'Harmattan, I998; Histoire et pouvoir en Europe médiane, A. Marès (dir.), L’Harmattan, 1997; Europe médiane. Aux sources des identités nationales, M. Delaperrière, B. Lory et A. Marès (dir.), Institut d'études slaves, 2005.

35. V. Čapek, « Podíl historie jako školní disciplíny na výchově k evropanství» («La part de l'histoire comme discipline scolaire dans l'éducation à l'européanité»), dans J. Raclavská (éd.), Česká a polská mládež o sobè. Młodzież polska i czeska o sobie (La jeunesse tchèque et polonaise sur elle-même), Ostrava, MŠMT České republiky a MEN Polské republiky, I999, p. 64. 
l'autre, se subdivise en ses composants qui se définissent en opposition les uns par rapport aux autres. Cela vaut même entre Slaves : définis par rapport aux Germains, ils se divisent essentiellement en Tchèques, Polonais et Slovènes, et par rapport aux Hongrois, en Slovaques, en Croates, et dans les divers groupes slaves orientaux qui vivent dans cette région. Ceux qui se définissent par rapport à tous les autres groupes, ce sont les Juifs.

Lélément juif est justement souvent devenu le symbole type de l'Europe centrale qui se trouverait partout où, en Europe, vivent des Juifs (selon Milan Kundera). L'écrivain serbe Danilo Kiš, parfaitement d'accord sur ce point avec Kundera, va encore plus loin : pour lui, les Juifs ont toujours représenté en Europe centrale le mouvement et le changement, ils ont constitué un trait d'union entre les petites nations européennes, et ils ont contribué dans une très large mesure au caractère multiculturel de cette partie de l'Europe ${ }^{36}$. L'histoire de l'Europe centrale a abouti à la destruction et à l'expulsion des Juifs de sorte que, selon Kišs, leur destin tragique reflète le traumatisme psychique subi par l'intellectuel centre-européen, arraché à son terreau "natal». D'autant plus que les écrivains d'origine juive ayant vécu en Europe centrale comme Franz Kafka, Bruno Schulz, Isaac Bashevis Singer, Elias Canetti, György Konrád, Sándor Márai et d'autres encore, ont souvent été qualifiés d'écrivains "centre-européens». Ce qui ne signifie pas que l'Europe centrale est nécessairement juive : le plus souvent, elle est aussi liée à la tradition chrétienne - le pape Jean-Paul II a par exemple parlé du christianisme comme valeur universelle d'intégration qui doit présider à l'unification politique et économique de l'Europe; et c'est justement l'Europe centrale qui, d'après lui, peut devenir l'exemple d'un dialogue interconfessionnel et interculturel entre croyants des religions chrétienne orientale et chrétienne occidentale, non seulement au sens où elles seraient pour ainsi dire "les deux poumons de l'Europe", mais aussi parce que c'est ici que s'interpénètrent harmonieusement et se complètent mutuellement le rite oriental (Cyrille et Méthode) et le rite occidental latin ${ }^{37}$. Dans cet ordre d'idées, l'un de ses prédécesseurs, Paul VI, a présenté la règle de saint Benoît comme le premier document écrit proclamant une constitution européenne et exigeant que les Européens adoptent un comportement commun, en l'occurrence un mode de vie chrétien $^{38}$. Le théologien et philosophe tchèque, Otakar A. Funda, rappelle pour

36. D. Kiš, Čas anatomije, Belgrade, Nolit, 1978 (en français : La Leçon d’anatomie, Fayard, 1998).

37. Voir S. Sowiński et R. Zenderowski, Europa droga Kościoła. Jan Paweł II o Europie i europejskości (L'Europe par la voie de l'Église. Jean-Paul II sur l'Europe et l'européanité), Wrocław, Ossolineum, 2003. 38. Ibid., p. 36. 
sa part que font partie des sources de la civilisation européenne moderne non seulement le judaïsme et le christianisme, mais aussi l'Antiquité et plus tard l'humanisme laïque ${ }^{39}$. La question de savoir si l'Europe a des racines religieuses ou mondaines, reste donc ouverte pour l'avenir et fera sans doute l'objet de discours abstraits et théoriques et d'interprétations idéologiques plutôt que d'une thèse admise par tous.

L'histoire littéraire parle parfois, elle aussi, d'écrivains qu'elle dit centreeuropéens ou d'auteurs liés à cet espace par leur langue et leur histoire personnelle et qui, dans leurs textes, expriment avec une intensité particulière les idées propres à la centre-européanité du fait que thématiquement, ces textes évoquent l'atmosphère culturelle de l'Europe centrale. En dépit des différences de génération et d'esthétique existant entre ces écrivains, on voit bien en effet que leurs œuvres sont liées ou se caractérisent par une genèse et une origine communes - les dernières décennies de l'existence de l'AutricheHongrie, c'est-à-dire son développement culturel sur le mode analogique, tel qu'il a succédé à l'éclatement de la monarchie en I9I8 pour prendre fin après 1930 dans les parties les plus diverses de l'ancien empire, comme Prague, Cracovie, Vienne, Bratislava, Košice, Budapest, Zagreb, Lubiana ${ }^{40} \ldots$ Bien que dans la vie politique, les États centre-européens se soient à cette époque strictement différenciés selon la nationalité et aient refusé toute forme de vie en commun, sur le front culturel on assiste au contraire à une remarquable symbiose dans l'ordre des -ismes, c'est-à-dire des mouvements et des courants artistiques, et ce en dépit du fait que ces ouvres reflètent de façon critique la situation de l'Autriche-Hongrie, présentée comme "prison des peuples». Malgré l'éclatement de celle-ci, c'est-à-dire de la monarchie danubienne dont Claudio Magris parle avec tant d'emphase, malgré l'essor des États successeurs parmi lesquels figure notamment la Tchécoslovaquie, le phénomène de centrisme centre-européen n'en a pas moins continué à fonctionner et à jouer son rôle. Il n'a pas été possible d'étouffer complètement le phénomène de l'Autriche-Hongrie qui a constitué une tentative - certes vaine, brutale et contraignante - de créer une situation de centrisme centre-européen relativement stable et équilibrée. C'est pourquoi une forme vague et «fluide» lui convient mieux que les limites fixes d'un État, même multinational et fédératif. Le transfert du conflit du cadre fédératif commun de la monarchie au niveau international à partir des années 1930 (il s'agit surtout de la montée du fascisme et de l'effondrement du système instauré par le traité de Versailles pour garantir l'équilibre centre-européen) conduit

39. O. A. Funda, Znavená Evropa umírá (L'Europe fatiguée meurt), Prague, Karolinum, 2000.

40. Voir A. Měšt'an, ouvr. cité, p. 369-376. 
après 1945 à ce que reproches et critiques à l'endroit de l'Autriche-Hongrie soient relayés par des tonalités nostalgiques et sentimentales.

À cette évolution est liée également la question de savoir s'il existe un style centre-européen et s'il est spécifique, c'est-à-dire la question de savoir si nous pouvons retrouver l'idée d'Europe centrale au niveau du genre, de la poétique et du style, donc au niveau des structures littéraires mêmes. Comme l'a montré l'ambitieux projet de génétique littéraire portant sur Les Métamorphoses génériques dans le contexte centre-européen (2003-20064I), cette spécificité se manifeste le plus souvent au niveau du grotesque, de l'ironie, de la satire, ou encore de l'essor des petites formes théâtrales du début du $\mathrm{Xx}^{\mathrm{e}}$ siècle sous les espèces d'un nouveau type de cabaret politique satirique et, plus généralement, au niveau de l'explosion des formes non traditionnelles de la culture populaire auparavant méprisée. Des théoriciens polonais et slovaques (Edward Kasperski, Tibor Žilka, etc.) considèrent par exemple à juste titre qu'à la fin du $\mathrm{XX}^{\mathrm{e}}$ siècle, on peut étudier ce type de discours (surtout au niveau de l'interpoéticité) comme des modèles et des constantes culturels supratemporels authentiques ${ }^{42}$. Ce n'est nullement un hasard si la variante du postmodernisme centre-européen, telle qu'on la rencontre dans les œuvres en prose volontiers grotesques et satiriques des écrivains contemporains polonais, ukrainiens, tchèques et slovaques, devient ici un objet digne de la recherche «sérieuse».

On voit bien que le phénomène qu'on appelle «Europe centrale» ne cessera pas, même en passant du $\mathrm{Xx}^{\mathrm{e}}$ au $\mathrm{XxI}^{\mathrm{e}}$ siècle, de faire l'objet d'un discours métacritique permanent qui mettra en émoi publicistes, politologues, mais aussi historiens de la littérature, écrivains et artistes en tout genre. L'histoire de l'Europe, donc l'histoire du centrisme centre-européen, en dépit de ses connotations négatives et douloureuses, ne peut s'effacer de la mémoire culturelle : on peut faire de l'Europe centrale un usage seulement sensible ou fonctionnel, la rassembler et la démembrer, s'en rapprocher et s'en éloigner, mais non l'oublier. Les méthodes politiques et administratives sont efficaces,

4I. L. Pavera et I. Pospišil, Žánrové metamorfózy v stréedoevropském kontextu (Métamorphoses génériques dans le contexte centre-européen), vol. I., Brno, Istenis, 2003; vol. 2. (Stabilita a labilita žánrů [Stabilité et labilité des genres]), L. Pavera (éd.), Opava, Slezská univerzita, 2005; vol. 3. (Žánry živé, mrtvé, revitalizované [Genres vivants, morts, revitalisés]), L. Pavera (éd.), Opava, Slezská univerzita, 2006.

42. E. Kasperski, «Związki literackie, intertekstuałność i literatura powszechna» («Relations littéraires, intertextualité et littérature générale»), dans D. Durišin (et al.), Medziliterárny centrizmus stredoeurópskych literatúr, ouvr. cité, p. I08-I30, et T. Žilka, Text a posttext, Nitra, Vysoká škola pedagogická, I995. 


\section{Recherches \& Travaux - $\mathrm{n}^{\circ} 80$}

rapides et fiables, mais ne durent pas; les méthodes économiques sont plus stables, mais ce qu'il y a de plus solide et de plus durable, ce sont seulement les archétypes psychiques et culturels qui se sont constitués au fil des siècles. Si l'Europe centrale comme espace spirituel et géographique a toujours été, sur le plan historique, une tentative pour instaurer un équilibre culturel entre Occident et Orient, il ne reste pour l'avenir qu'à espérer que les réflexions quant à sa forme ne chercheront pas à constituer une alternative politique réelle à la coexistence dans le dialogue, et qu'elle restera un exemple de cohabitation tolérante et de respect de la pluralité dans l'unité, mais surtout un lieu de débat et de connaissance réciproque. 